\title{
Paired Associative Stimulation of the Temporal Cortex: Effects on the Auditory Steady-State Response
}

\author{
Sarah Engel*, Robert Daniel Heinrich Markewitz, Berthold Langguth \\ and Martin Schecklmann
}

Department of Psychiatry and Psychotherapy, University of Regensburg, Regensburg, Germany

Background: Paired associative stimulation (PAS) is the repeated combination of a sensory stimulus with transcranial magnetic stimulation (TMS) in close temporal association. Recently, a study demonstrated that PAS of an auditory stimulus together with TMS of the temporal cortex is capable of changing the amplitude of auditory evoked potentials (AEP).

Objective: This study examined the influence of tone duration and habituation in tem-

OPEN ACCESS

Edited by:

Paul Croarkin,

Mayo Clinic Minnesota,

United States

Reviewed by:

Jonathan Chia-Ho Lee,

Centre for Addiction and Mental

Health, Canada

Jennifer Rachel Goldschmied,

University of Pennsylvania,

United States

*Correspondence:

Sarah Engel

sarah@engelvita.de

Specialty section:

This article was submitted to Neuroimaging and Stimulation,

a section of the journal

Frontiers in Psychiatry

Received: 06 July 2017

Accepted: 24 October 2017

Published: 08 November 2017

Citation:

Engel S, Markewitz RDH, Langguth $B$ and Schecklmann M (2017) Paired

Associative Stimulation of the Temporal Cortex: Effects on the Auditory Steady-State Response.

Front. Psychiatry 8:227. doi: 10.3389/fpsyt.2017.00227 poral cortex PAS as elicited by 40 and $20 \mathrm{~Hz}$ amplitude modulated auditory steady-state responses (aSSR).

Methods: Eighteen subjects participated in two experiments, including two PAS protocols each, which consisted of 200 auditory stimuli $(4 \mathrm{kHz})$ paired with temporal cortex TMS with an interstimulus interval (ISI) of $45 \mathrm{~ms}$ between tone onset and TMS pulse, delivered at $0.1 \mathrm{~Hz}$. Experiment 1 compared auditory stimuli with different lengths [PAS (23 ms) vs. PAS (400 ms)]. Experiment 2 investigated verum vs. sham PAS. aSSR for the paired tone $(4 \mathrm{kHz})$ and a control tone $(1 \mathrm{kHz})$ were measured pre- and post-interventional-using $40 \mathrm{~Hz}$ aSSR in experiment 1 and both 20 and $40 \mathrm{~Hz}$ aSSR in experiment 2.

Results: A statistically significant, sham-controlled decrease in amplitude was observed for the $20 \mathrm{~Hz}$ aSSR using the $4 \mathrm{kHz}$ PAS carrier frequency in experiment 2.

Conclusion: Frequency-specific effects for the $20 \mathrm{~Hz}$ aSSR confirm the feasibility of auditory PAS and highlight the secondary auditory cortex as its target site, introducing new possible treatment protocols for patients suffering from tinnitus. The amplitude decrease can be explained by principles of spike timing-dependent plasticity and the superposition model of aSSR.

Keywords: paired associative stimulation, auditory steady-state response, temporal cortex, tinnitus, spike-timing dependent plasticity

\section{INTRODUCTION}

Transcranial magnetic stimulation (TMS) is a non-invasive stimulation technique which uses a coil placed on the scalp to apply magnetic stimulation to possible target areas of the cortex (1). A series of TMS pulses is called repetitive TMS (rTMS), which can induce changes of excitability via processes similar to long term potentiation (LTP) and long term depression (LTD) (2). Paired associative 
stimulation (PAS) is the pairing of external sensory stimuli with TMS pulses applied to the corresponding cortical region of the peripheral stimulus capable of inducing changes in neuroplasticity (3). Based on the concept of spike timing-dependent plasticity (STDP), the effects of PAS depend strongly on the order of the cortical processing of the peripheral stimulus and the TMS pulse. If cortical neurons are stimulated post-synaptically with TMS before they are excited pre-synaptically by the sensory stimulus, synaptic connectivity is reduced via LTD-like effects (3). If this order is reversed, LTP-like effects are expected (3). A recent pilot study revealed that the principles of PAS apply not only to the motor cortex (4) and the primary somatosensory cortex (5) but to the human secondary auditory cortex as well (6).

Tones of a specific carrier frequency can have sinusoidally modulated sound levels. These amplitude modulated tones (AM) are used to evoke auditory steady-state responses (aSSR) in the auditory cortex (7). They are recorded in the electroencephalogram as sinusoidal waves of the same frequency as the frequency of the amplitude modulation of the tone (8). So far there is no complete understanding of the mechanism underlying the aSSR. In theory, depending on the modulation frequency different parts of the auditory cortex can be activated and the generated neural responses are thought to correspond with those of transient auditory evoked potentials (AEP) (7). For example, 40-Hz AM aSSR have a modulated sound level with a period of $25 \mathrm{~ms}$. Therefore, the $40-\mathrm{Hz}$ AM aSSR most likely correlates with the Pa-component, a middle latency AEP with a latency of about $25 \mathrm{~ms}$ (9-12). There is a lot of evidence that the source of the $40 \mathrm{~Hz}$ aSSR is localized in the Heschl's gyrus, which is considered to be the primary auditory cortex (13-15), which is also be presumed to be the origin of the Pa-component (16). Equivalent to the $40 \mathrm{~Hz}$ AM tone, a $20-\mathrm{Hz}$ aSSR may reflect the P1-component, a late AEP with a latency of $50 \mathrm{~ms}$ generated in the secondary auditory cortex (16).

A pilot study showed that PAS of the auditory cortex is capable to induce timing- and tone-specific inhibitory effects as indicated by amplitude decreases of long-latency AEP (6). PAS (45 ms) showed greater decreases than PAS (10 ms) [PAS protocol with an interstimulus interval (ISI) of $45 \mathrm{~ms}$ between tone onset and TMS pulse vs. a PAS protocol with an ISI of $10 \mathrm{~ms}$ ] (6). Schecklmann et al. assumed that the auditory evoked signal reaches the secondary auditory cortex, which has been stimulated with TMS, after about $50 \mathrm{~ms}$ (6). Thus, the more pronounced amplitude reduction after PAS (45 ms) was interpreted as a consequence of the shorter interval between pre- and postsynaptic excitation as compared to PAS (10 ms) (6). The effects seemed also to be frequency specific, as the amplitude decrease was more pronounced for the $4 \mathrm{kHz}$ tone which had been used for the PAS intervention in contrast to a $1 \mathrm{kHz}$ control tone (6). No significant effects on the AEP were observed after 0.1 or $1 \mathrm{~Hz}$ rTMS without acoustic stimulation that were used as control conditions (6). In this pilot study, the paired tone had a duration of $400 \mathrm{~ms}$ which represents a relatively long duration as PAS of the somatosensory or motor system uses electric stimuli in the range of microseconds (3). Thus, the long duration might have contributed to the inhibitory effect. One further limitation of the pilot study was the lack of a control condition that consisted of auditory stimulation in combination with sham stimulation (6). Therefore, habituation effects induced by numerous repetitions of the presented tones could not be ruled out as a potential confounder, even if the timing-specific effects (same number of presented acoustic stimuli) argued against pure habituation effects as an explanation for the observed amplitude decreases (6). Furthermore, only effects on the secondary auditory cortex were evaluated by assessing late AEP (6).

The aims of the present work were to control for effects of the duration of the paired auditory stimulus and for unspecific effects such as habituation. For this purpose, we conducted two experiments contrasting long- and short PAS tones (experiment 1) and verum (using a defined stimulation intensity) and sham stimulation (experiment 2) for the PAS stimulation. Effects were measured via aSSR using $40 \mathrm{~Hz}$ amplitude (experiment 1) and both 40 and $20 \mathrm{~Hz}$ amplitude modulation (experiment 2). Therefore, effects on the primary ( $40 \mathrm{~Hz}$ AM aSSR) and on the secondary (20 Hz aSSR) auditory cortex can be evaluated.

We hypothesized that PAS of the temporal cortex can induce changes in neuroplasticity. According to the model of STDP the chosen ISI of $45 \mathrm{~ms}$ between tone onset and the TMS pulse will lead to an increase in amplitude of the $40 \mathrm{~Hz}$ AM aSSR representing the primary auditory cortex and to a decrease in amplitude of the $20 \mathrm{~Hz}$ AM aSSR representing the secondary auditory cortex (16).

\section{MATERIALS AND METHODS}

\section{Subjects and Recruitment}

Eighteen students from the University of Regensburg participated in the study. We recruited all subjects by word of mouth. All volunteers received a monetary compensation and had no relevant neurological or medical disorders. Seventeen subjects completed a multiple choice vocabulary test ("Mehrfach-WortschatzIntelligenztest", third edition, MWT-B) (17). Participant 18 was excluded from this test as she was not a German native speaker. Hearing function was assessed by pure tone audiometry testing seven frequencies between $125 \mathrm{~Hz}$ and $8 \mathrm{kHz}$ (Midimate 622D, Madsen Electronics, GN Otometry, Denmark). All participants had a hearing threshold below $30 \mathrm{~dB}$ HL for all tested frequencies. All subjects gave written informed consent after being informed about contraindications, side effects (3), and study procedure. The study was approved by the Ethics Committee of the University of Regensburg and performed in accordance with the last revision of the Declaration of Helsinki.

\section{General Study Procedure}

All participants completed four different sessions of PAS, two within each experiments. We (Sarah Engel and Robert Daniel Heinrich Markewitz) conducted the experiments in a quiet room of the Department of Psychiatry and Psychotherapy of the University of Regensburg at the Bezirksklinikum Regensburg. One of us operated the TMS stimulator, while the other one overviewed the stimulus presenting computer program. Within experiment 1 and 2, we presented the different PAS-conditions in a randomized order 1 week apart with a 6-month interval between experiments 1 and 2. We measured aSSR before and after each PAS-condition (see Figure 1). 


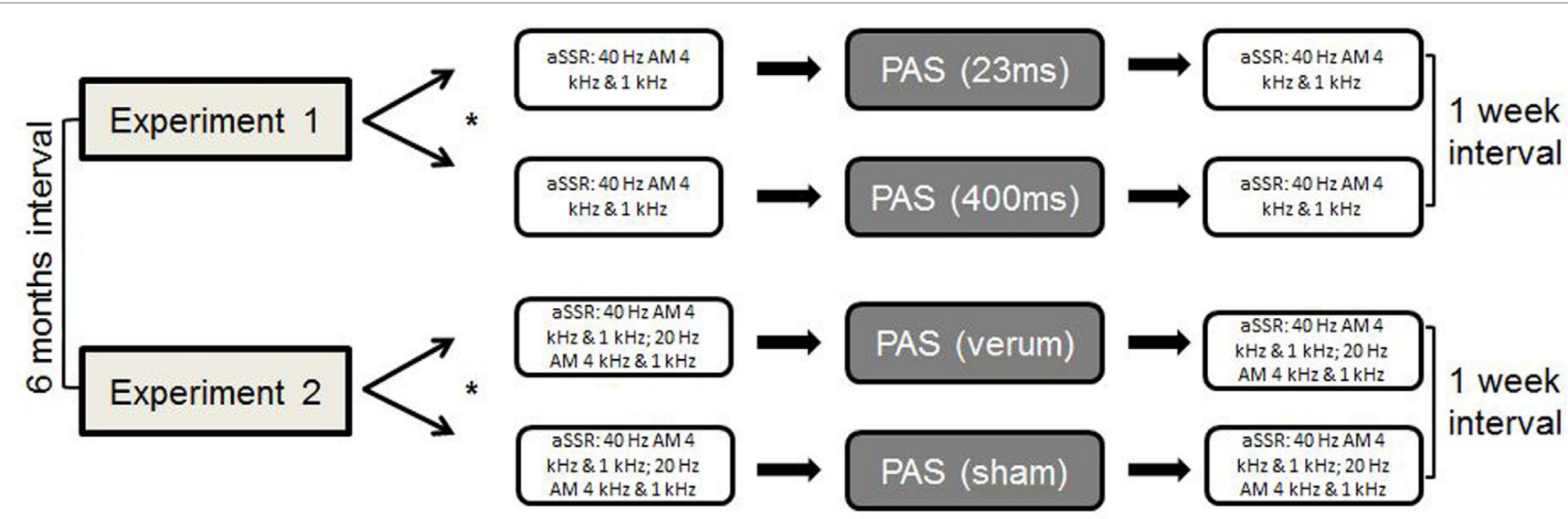

*randomised order of PAS conditions

FIGURE 1 | General study procedure.

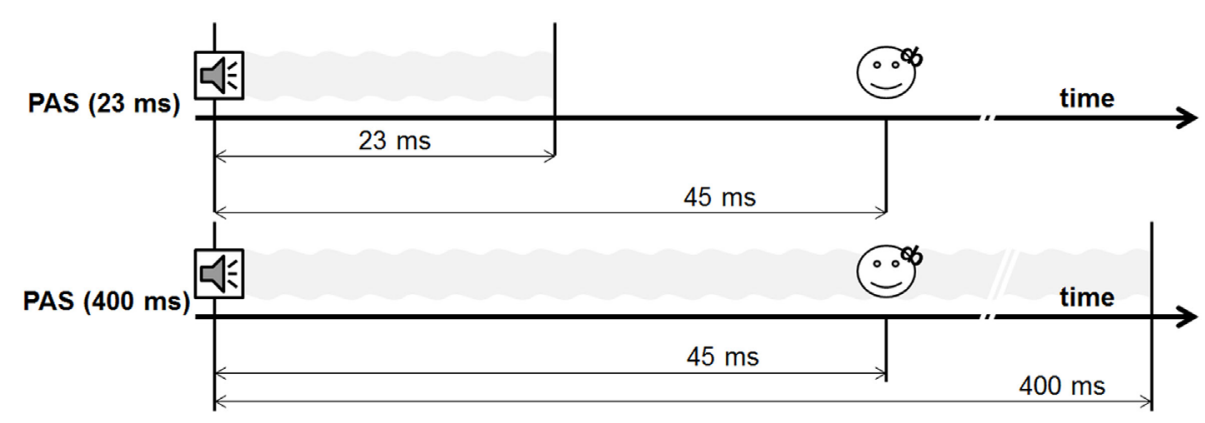

FIGURE 2 | Design experiment 1.

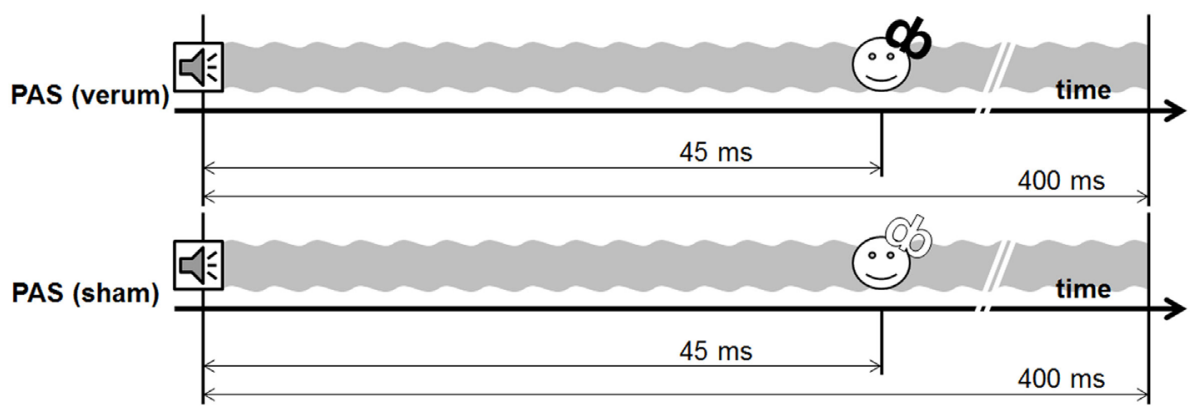

FIGURE 3 | Design experiment 2.

In the first session of each experiment, we determined the stimulation intensity [110\% resting motor threshold (RMT)] for each subject following the protocol of Schecklmann and colleagues $(6,18)$.

For each experiment, we evaluated the sensation levels for the tones used during the experiments using Adobe audition 3.0 (Adobe Systems, DE, USA). We presented all tones binaurally through inserted earphones (E-A-RLINK, Foam Eartips for Insert Earphones, 3M, E-A-R, Etymotic Research, Inc.) at $60 \mathrm{~dB}$ sensation level.

\section{PAS Protocols}

All PAS protocols lasted around $33 \mathrm{~min}$ and consisted of 200 stimulus pairs of an auditory stimulus of $4 \mathrm{kHz}$ and a TMS pulse with an ISI of $45 \mathrm{~ms}$ presented with a stimulation frequency of $0.1 \mathrm{~Hz}$. We used an ISI of $45 \mathrm{~ms}$ as the pilot study showed the largest effects for this condition (6).

During experiment 1 , we performed two different PAS protocols, using a $400-\mathrm{ms}$ tone and a $23-\mathrm{ms}$ tone [PAS ( $23 \mathrm{~ms}$ ) vs. PAS (400 ms)] (see Figures 2 and 3). The shortest tone length enabling 
a pure tone percept was $23 \mathrm{~ms}$ as evaluated by subjective judgment and fourier analysis as implemented in Adobe Audition.

Experiment 2 included a sham and a verum PAS protocol, using a $400-\mathrm{ms}$ tone [PAS (sham) vs. PAS (verum)]. The PAS (verum) protocol was the same protocol as the PAS (400 ms) protocol used in experiment 1 . In the sham condition, the watercooled figure of eight coil was reverted in a way that the back of the coil was directed to the head of the subject. The magnetic field is decreased to one-sixth on this side of the coil as indicated by own measurements [compare technique in van Doren et al. (19)]. This sham condition guaranteed comparable sensations with respect to the click sound and the vibration of the coil.

We placed the coil over the left auditory cortex following the protocol of the pilot study (6) by using a standard procedure based on EEG coordinates (20). The TMS pulses were presented with a water-cooled figure of eight coil (MAGPRO, Medtronic, USA, outer diameter: $90 \mathrm{~mm}$; water-cooled double coil). The computer software Presentation (Neurobehavioral Systems, Inc., USA) triggered the TMS pulse and presented the auditory stimuli. To ensure the exact timing of the ISI ( $45 \mathrm{~ms}$ ), we measured the acoustic stimuli from the insert earplugs with a sound-level meter linked to one channel of the EEG amplifier and the TMS artifact which is induced by stimulation of the electrode cap.

\section{aSSR Recording and Measurement}

We recorded aSSR with an EEG cap (Braincap Fast'n Easy $64 \mathrm{Ch}$ for TMS, Standard Layout, Easycap, Germany), reduced impedances below $10 \mathrm{k} \Omega$. We sampled EEG data with a frequency of $500 \mathrm{~Hz}$ (BrainAmp MR plus, Germany). We used AM tones of $800 \mathrm{~ms}$ duration (rise- and fall time: $75 \mathrm{~ms}$ ) with a carrier frequency of $4 \mathrm{kHz}$ (paired tone) or $1 \mathrm{kHz}$ (control tone), presented in a randomized order with a variable ISI $(2,800-3,200 \mathrm{~ms})$. For recording EEG, we used BrainVision (Brain Products $\mathrm{GmbH}$, Germany).

\section{Experiment 1}

In experiment 1 , we compared two PAS protocols, one using a $23-\mathrm{ms}$ tone of $4 \mathrm{kHz}$ [PAS (23 ms)] and the other one using a $400 \mathrm{~ms}$ tone of $4 \mathrm{kHz}$ [PAS (400 ms)]. As read-out parameter we used $40 \mathrm{~Hz}$ AM aSSR, measured as described above, in order to evaluate the effects on the primary auditory cortex. We used two different carrier frequencies: a $4-\mathrm{kHz}$ tone, correlating with the $4 \mathrm{kHz}$ we used during the PAS intervention (paired tone), and a $1-\mathrm{kHz}$ tone (control tone). The aSSR measurments before and after the PAS intervention lasted about 7.5 min each.

\section{Experiment 2}

In experiment 2, we compared a verum condition (actual stimulation of the auditory cortex) with a sham condition [PAS (verum) vs. PAS (sham)]. As in experiment 1 , we also used a $400-\mathrm{ms}$ tone with a carrier frequency of $4 \mathrm{kHz}$ for the PAS intervention. In order to evaluate the effects on the primary and secondary cortex, we measured 20 and $40 \mathrm{~Hz}$ AM aSSR before and after the intervention with two different carrier frequencies, 4 (paired tone) and $1 \mathrm{kHz}$ (control tone). Accordingly, four acoustic stimuli were presented ( $40 \mathrm{~Hz}$ AM aSSR of $4 \mathrm{kHz}$ carrier frequency, $40 \mathrm{~Hz}$ AM aSSR of $1 \mathrm{kHz}$ carrier frequency, $20 \mathrm{~Hz}$ AM aSSR of a $4 \mathrm{kHz}$ carrier frequency, and $20 \mathrm{~Hz} \mathrm{AM}$ aSSR of $1 \mathrm{kHz}$ carrier frequency). Measurements of the aSSR lasted about 15 min each.

\section{Data Analysis and Statistical Evaluation}

We transferred all recorded EEG data to EEGLAB (21), created epochs of $4.5 \mathrm{~s}$ (from $2 \mathrm{~s}$ before till $2.5 \mathrm{~s}$ after tone onset), and processed the EEG data using a high $(0.1 \mathrm{~Hz})$ and a low $(90 \mathrm{~Hz})$ pass filter. After visual inspection, we excluded segments containing muscle artifacts, electrodes with signal loss, and segments with strong background noise. Further artifacts were rejected using independent component analysis.

After a subsequent visual inspection for any remaining artifacts, we interpolated the EEG data and re-referenced it to an average reference. The electrode $\mathrm{FC} z$, which was used as a reference electrode during measurements, was reconstructed. EEG channels which were omitted before due to artifacts were then reconstructed using surrounding electrodes for interpolation purposes.

For the analysis of the $20-\mathrm{Hz}$ aSSR, we filtered the data with $18-22 \mathrm{~Hz}$, while a filter of $38-42 \mathrm{~Hz}$ was used for analysis of the $40-\mathrm{Hz}$ aSSR.

After manually inspecting all segments of each participant for artifacts, we identified 59 as the minimum number of segments, i.e., the measurement with the smallest number of remaining segments counted 59 segments. Therefore, we used the first 59 trials of each participant and of each condition for further calculations.

Then we transferred the EEG data to FieldTrip (21). We calculated and rectified the mean voltage of all trials. Thereafter, we performed a baseline correction for the interval of $300 \mathrm{~ms}$ before the tone onset. We inspected the averaged and rectified trials for plausibility using topographies and trajectories. We decided to use time-locked data (averaging of the single segments) and evoked activity as the principle of STDP is related to an exact and constant timing of two stimuli.

For further statistical analysis and based on plausibility checks, we chose a time of interest of 500-800 ms to avoid interference with long-latency AEP. Our region of interest was in the frontoparietal area (F1, Fz, F2, FC1, FCz, FC2, C1, Cz, C2). We extracted the data from these electrodes and imported it into SPSS 18.0.0 (SPSS, USA).

We computed $2 \times 2$ analyses of variance with two withinsubjects factors "time" (pre vs. post) and "PAS-condition" (experiment 1: short vs. long tone; experiment 2: sham vs. verum condition), for both tones ( $1 \mathrm{kHz}$ control tone and $4 \mathrm{kHz}$ paired tone) and both types of AM tones in experiment $2(20 \mathrm{~Hz} A M$ and $40 \mathrm{~Hz} \mathrm{AM}$ ). We used a two tailed paired Student's $t$-test for post hoc analysis for statistically significant interaction effects. We performed corrections for multiple comparisons using Bonferroni correction.

\section{RESULTS}

All participants had a mean age of 21.28 years $[ \pm 2.37$ standard deviation (SD)] with an age range from 19 to 28 years. All participants were right handed, 10 were female. The mean hearing level $(\mathrm{dB} H \mathrm{HL})$ was $13.318 \pm 2.572 \mathrm{SD}$ with a range $(\mathrm{dB} H \mathrm{HL})$ of 8.890-17.78. There was no significant difference between the 
RMT for experiment 1 and $2(T=-0.414 ; d f=1 ; 17 ; p=0.684)$. Experiment 1 and 2 were completed by all 18 participants without any reports of side effects.

\section{Explanation of Plausibility}

For all subjects, we could identify the typical topography of both the 20 - and the $40-\mathrm{Hz}$ aSSRs as a positive maximum in the frontocentral region for the sensitive interval of 500-800 ms including the electrodes: F1, Fz, F2, FC1, FCz, FC2, C1, Cz, C2.

\section{Effects of PAS Adjusted for Multiple Comparisons (Bonferroni)}

Experiment 1 showed no significant effects for the $1 \mathrm{kHz}$ tone (all $F$-values $<5.375$; all $p$-values $>0.066)$ and no significant effect for the $4 \mathrm{kHz}$ tone (all $F$-values $<2.232$; all $p$-values $>0.306$ ).

Looking at the results of experiment 2 , we found a significant result for the $20 \mathrm{~Hz}$ AM $4 \mathrm{kHz}$ paired tone for the main effect PAS condition $(F=8.816 ; d f=1 ; 17 ; p=0.018)$ as well as for the interaction "time by PAS condition" $(F=6.11 ; d f=1 ; 17 ; p=0.048)$ (see Figure 4), but not for the time main effect $(F=0.167$; $d f=1 ; 17 ; p=1.376)$. The post hoc paired Student's $t$-test showed a significant decrease in the amplitude from pre to post stimulation for the verum condition $(t=3.505 ; d f=1 ; 17 ; p=0.012)$ but no significant decrease in amplitude of the verum condition in contrast to the sham condition after the stimulation $(t=2.120$; $d f=1 ; 17 ; p=0.196)$. For the sham condition there were no differences between the pre- and post-measurement $(t=0.253$; $d f=1 ; 17 ; p=1.606)$. Before stimulation verum and sham differed not significant $(t=-1.374 ; d f=1 ; 17 ; p=0.374)$. Therefore, a significant decrease of the $20 \mathrm{~Hz}$ AM $4 \mathrm{kHz}$ tone-which was paired in the PAS - could be observed for the verum condition with no changes for the sham condition. There were no significant effects for the $40 \mathrm{~Hz}$ AM $4 \mathrm{kHz}$ tone (all $F$-values $<1.521$, all $p$-values > 0.468). All effects for both 40 and $20 \mathrm{~Hz} \mathrm{AM}$ tones with the carrier frequency of $1 \mathrm{kHz}$ were not significant (all $F$-values $<2.188$; all $p$-values $>0.314$ ).

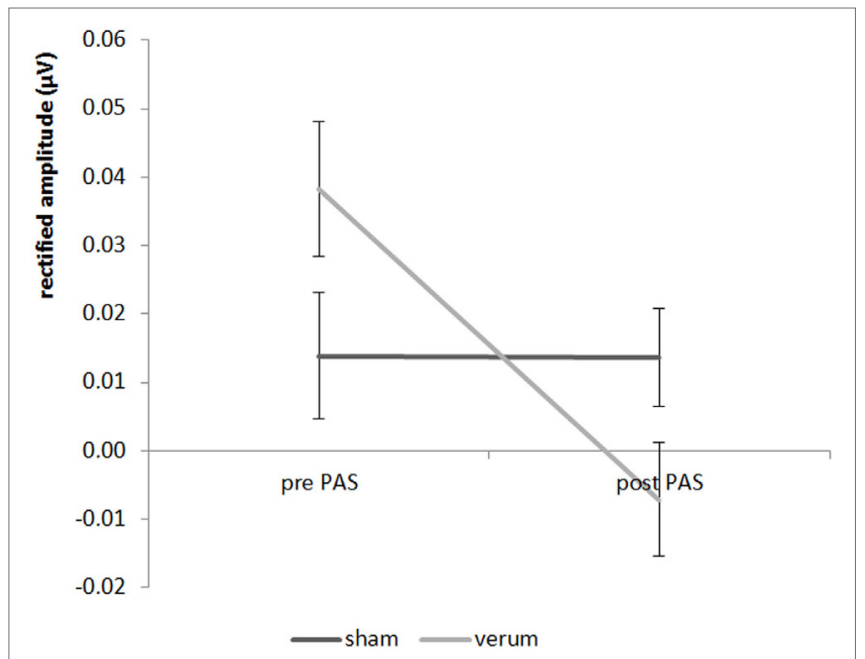

FIGURE 4 | Results for the $20 \mathrm{~Hz}$ AM $4 \mathrm{kHz}$ paired tone in experiment 2 ( \pm standard deviation).

\section{DISCUSSION}

The main finding of our experiments was a significant interaction effect showing a sham-controlled PAS induced decrease of the $20 \mathrm{~Hz}$ aSSR amplitude. This effect was frequency specific as it occurred only for the $4 \mathrm{kHz}$ tone (carrier frequency which was used for pairing in the PAS) but not for the $1 \mathrm{kHz}$ control tone. We could not find any statistically significant results for the $40 \mathrm{~Hz}$ aSSR, neither for the $1 \mathrm{kHz}$ nor for the $4 \mathrm{kHz}$ carrier frequency, including experiment 1 (short tone vs. long tone) and 2 (verum vs. sham condition). The significant frequency-specific interaction effect may support the notion that PAS with combined auditory and TMS induces an inhibitory mechanism by inducing STDP. A pure habituation effect can be excluded as auditory stimulation combined with sham TMS (experiment 2) did not induce a significant amplitude reduction of the aSSR. The observed frequency specificity is in line with the results of the pilot study (6), where inhibitory effects were also observed primarily for the frequency of the tone that was paired with TMS in the PAS protocol. The frequency specificity is a further argument for the assumption that combined auditory stimulation and TMS is critical for the observed inhibitory effects.

The reduction of the $20 \mathrm{~Hz}$ aSSR after PAS fits well with superposition theory which explains the generation of aSSR (9-12). Based on the theory of STDP $(2,3)$ the PAS protocol with an ISI of $45 \mathrm{~ms}$ should lead to LTD-like effects for external stimuli which arrive in the stimulated cortical area later than $45 \mathrm{~ms}$ after auditory stimulation. Under the assumption that the $20 \mathrm{~Hz}$ aSSR is generated by superposition of the P1/P50 (which has a latency of $50 \mathrm{~ms}$ ), effects of a PAS ( $45 \mathrm{~ms}$ ) protocol should lead to amplitude decrease which was the case in the present study.

There were no significant effects for the $40 \mathrm{~Hz}$ aSSR neither in experiment 1 nor in experiment 2 . As shown in the plausibility check, we were able to evoke the $40 \mathrm{~Hz}$ aSSR, but the PAS protocols did not induce any changes of the amplitude of the $40 \mathrm{~Hz}$ aSSR. $40 \mathrm{~Hz}$ aSSR are presumably generated by the primary auditory cortex (12), whereas the $20 \mathrm{~Hz}$ aSSR are most likely generated in the secondary cortex (16). Therefore, the significant decrease in amplitude of the $20 \mathrm{~Hz}$ aSSR as compared to no significant change in amplitude for the $40 \mathrm{~Hz}$ aSSR might be explained by the different anatomical origins of the 20 - and the $40-\mathrm{Hz}$ aSSR as described above. While the primary auditory cortex occupies most of the Heschl's gyrus deep in the Sylvian fissure (22), the secondary auditory cortex (22), lies next to the primary auditory cortex on the external surface of the cortex. Due to its superficial location the secondary auditory cortex can be better reached with TMS than the primary auditory cortex. The individual stimulation intensity for the PAS intervention was determined as $110 \%$ of the RMT and, therefore, depended on the anatomy of the motor cortex, which lies as part of the precentral gyrus on the outer surface of the cortex as well (22). As such, we assumed that a $10 \%$ increase of the RMT will also be able to reach the auditory cortex. However, whether the stimulation intensity is high enough to have a direct effect on the primary auditory cortex is questionable. Since effects on the secondary auditory cortex could be observed, but none on the primary auditory cortex, we can assume that the intensity level of $110 \%$ of the RMT, we used 
during the intervention, may not be high enough to induce direct changes in neuroplasticity in the primary auditory cortex. For further experiments, we should take into account that the intensity of the electromagnetic field is inversely proportional to the distance from the TMS-coil (3).

Notably, we cannot exclude that TMS effects propagate from the secondary to the primary auditory cortex (23). However, such transsynaptically propagated effects on the primary auditory cortex would be too late to induce any STDP like effects in the investigated PAS protocols. Because we did not see any statistically significant results for the $40 \mathrm{~Hz}$ aSSRs neither in experiment 1 nor in experiment 2, we cannot draw any conclusions about the influence of different tone lengths of the paired tone. Further experiments using $20 \mathrm{~Hz}$ aSSR as read-out parameter will be needed to investigate the relevance of the tone length of the paired tone. Moreover, further experiments investigating the impact of different PAS intervals would be useful to confirm STDP as the underlying mechanism for the observed results.

Further experiments will also be necessary for additional evaluation of tonotopical effects of PAS on aSSR (e.g., using different frequencies as different stimulation conditions for the tones paired with the TMS-pulse during the intervention). Only then can an assessment of the potential of PAS as a tool both for research purposes and treatment of medical conditions be undertaken. For instance, PAS could be applied therapeutically to attenuate tinnitus symptoms. Pathogenesis of tinnitus, a phantom perception of sound (24), is thought to originate from abnormal neural activity $(24,25)$ and a decrease of functional inhibiting pathways (26). Our study contributes to the findings of the previous study (6) that depending on timing PAS is capable of inhibiting neural activities in the auditory cortex. Therefore, PAS of the auditory cortex could be used to reduce abnormal neural activity and to compensate the missing inhibiting pathways found in patients with tinnitus. If PAS proves to have a strictly tonotopical effect on aSSR it might even offer an individualized therapy option for people

\section{REFERENCES}

1. Hallett M. Transcranial magnetic stimulation: a primer. Neuron (2007) 55(2):187-99. doi:10.1016/j.neuron.2007.06.026

2. Pascual-Leone A, Valls-Sole J, Wassermann EM, Hallett M. Responses to rapid-rate transcranial magnetic stimulation of the human motor cortex. Brain (1994) 117(Pt 4):847-58. doi:10.1093/brain/117.4.847

3. Siebner HR, Ziemann U. Das TMS-Buch: Handbuch der transkraniellen Magnetstimulation. Heidelberg: Springer Medizin (2007).

4. Stefan K, Kunesch E, Cohen LG, Benecke R, Classen J. Induction of plasticity in the human motor cortex by paired associative stimulation. Brain (2000) 123(Pt 3):572-84. doi:10.1093/brain/123.3.572

5. Wolters A, Sandbrink F, Schlottmann A, Kunesch E, Stefan K, Cohen LG, et al. A temporally asymmetric Hebbian rule governing plasticity in the human motor cortex. J Neurophysiol (2003) 89(5):2339-45. doi:10.1152/jn.00900.2002

6. Schecklmann M, Volberg G, Frank G, Hadersdorfer J, Steffens T, Weisz N, et al. Paired associative stimulation of the auditory system: a proof-of-principle study. PLoS One (2011) 6(11):e27088. doi:10.1371/journal.pone.0027088

7. Santarelli R, Maurizi M, Conti G, Ottaviani F, Paludetti G, Pettorossi VE. Generation of human auditory steady-state responses (SSRs). II: addition of responses to individual stimuli. Hear Res (1995) 83(1-2):9-18. doi:10.1016/ 0378-5955(94)00185-S

8. Müller N, Schlee W, Hartmann T, Lorenz I, Weisz N. Top-down modulation of the auditory steady-state response in a task-switch paradigm. Front Hum Neurosci (2009) 3:1. doi:10.3389/neuro.09.001.2009 with tonal tinnitus, who could be treated with an inhibitory PAS intervention using their individual tinnitus pitch as a paired tone, other than rTMS which has shown moderate effects lasting from weeks to several months $(27,28)$. The potential therapeutic value of PAS in this context remains speculative.

\section{ETHICS STATEMENT}

This study was carried out in accordance with the recommendations of the last revision of the Declaration of Helsinki with written informed consent from all subjects. All subjects gave written informed consent in accordance with the Declaration of Helsinki. The protocol was approved by the Ethics Committee of the University of Regensburg.

\section{AUTHOR CONTRIBUTIONS}

All authors conceived and designed the research, as well as interpreted the results of the experiments. SE and RM performed the experiments. SE drafted the manuscript and prepared figures. MS and BL edited and revised the manuscript. All authors approved the final version of the manuscript.

\section{ACKNOWLEDGMENTS}

The authors would like to thank all participants and Dr. Larry Roberts for special advice on auditory steady-state responses.

\section{SUPPLEMENTARY MATERIAL}

The Supplementary Material for this article can be found online at http://www.frontiersin.org/article/10.3389/fpsyt.2017.00227/ full\#supplementary-material.

9. Plourde G, Stapells DR, Picton TW. The human auditory steady-state evoked potentials. Acta Otolaryngol Suppl (1991) 491:153-9; discussion 160. doi:10.3109/00016489109136793

10. Gutschalk A, Mase R, Roth R, Ille N, Rupp A, Hahnel S, et al. Deconvolution of $40 \mathrm{~Hz}$ steady-state fields reveals two overlapping source activities of the human auditory cortex. Clin Neurophysiol (1999) 110(5):856-68. doi:10.1016/ S1388-2457(99)00019-X

11. Suzuki T, Kobayashi K, Umegaki Y. Effect of natural sleep on auditory steady state responses in adult subjects with normal hearing. Audiology (1994) 33(5):274-9. doi:10.3109/00206099409071887

12. Presacco A, Bohorquez J, Yavuz E, Ozdamar O. Auditory steady-state responses to $40-\mathrm{Hz}$ click trains: relationship to middle latency, gamma band and beta band responses studied with deconvolution. Clin Neurophysiol (2010) 121(9):1540-50. doi:10.1016/j.clinph.2010.03.020

13. Brugge JF, Nourski KV, Oya H, Reale RA, Kawasaki H, Steinschneider M, et al. Coding of repetitive transients by auditory cortex on Heschl's gyrus. J Neurophysiol (2009) 102(4):2358-74. doi:10.1152/jn.91346.2008

14. Godey B, Schwartz D, de Graaf JB, Chauvel P, Liegeois-Chauvel C. Neuromagnetic source localization of auditory evoked fields and intracerebral evoked potentials: a comparison of data in the same patients. Clin Neurophysiol (2001) 112(10):1850-9. doi:10.1016/S1388-2457(01)00636-8

15. Bidet-Caulet A, Fischer C, Besle J, Aguera P, Giard M, Bertrand O. Effects of selective attention on the electrophysiological representation of concurrent sounds in the human auditory cortex. J Neurosci (2007) 27(35):9252-61. doi:10.1523/JNEUROSCI.1402-07.2007 
16. Eckert J, Lang N, Maurer K. Praxis der evozierten Potentiale: SEP, AEP, MEP, VEP; mit 60 Tabellen. 2nd ed. Darmstadt: Steinkopff (2005).

17. Lehrl S. Manual zum MWT-B. 5th ed. Balingen: Spitta-Verl (2005).

18. Pridmore S, Fernandes Filho JA, Nahas Z, Liberatos C, George MS. Motor threshold in transcranial magnetic stimulation: a comparison of a neurophysiological method and a visualization of movement method. JECT (1998) 14(1):25-7. doi:10.1097/00124509-199803000-00004

19. van Doren J, Langguth B, Schecklmann M. Electroencephalographic effects of transcranial random noise stimulation in the auditory cortex. Brain Stimul (2014) 7:807-12. doi:10.1016/j.brs.2014.08.007

20. Langguth B, Zowe M, Landgrebe M, Sand P, Kleinjung T, Binder H, et al. Transcranial magnetic stimulation for the treatment of tinnitus: a new coil positioning method and first results. Brain Topogr (2006) 18(4):241-7. doi:10.1007/s10548-006-0002-1

21. Delorme A, Makeig S. EEGLAB: an open source toolbox for analysis of single-trial EEG dynamics including independent component analysis. J Neurosci Methods (2004) 134(1):9-21. doi:10.1016/j.jneumeth.2003.10.009

22. Benninghoff A, Drenckhahn D. Taschenbuch Anatomie. 1st ed. München: Elsevier, Urban \& Fischer (2008).

23. Gueguin M, Le Bouquin-Jeannes R, Faucon G, Chauvel P, Liegeois-Chauvel C. Evidence of functional connectivity between auditory cortical areas revealed by amplitude modulation sound processing. Cereb Cortex (2006) 17(2):304-13. doi:10.1093/cercor/bhj148

24. Møller AR, Langguth B, de Ridder D, Kleinjung T. Textbook of Tinnitus. New York, NY: Springer New York (2011).
25. Norena AJ, Eggermont JJ. Changes in spontaneous neural activity immediately after an acoustic trauma: implications for neural correlates of tinnitus. Hear Res (2003) 183(1-2):137-53. doi:10.1016/S0378-5955(03)00225-9

26. Roberts LE, Moffat G, Baumann M, Ward LM, Bosnyak DJ. Residual inhibition functions overlap tinnitus spectra and the region of auditory threshold shift. J Assoc Res Otolaryngol (2008) 9(4):417-35. doi:10.1007/s10162-008-0136-9

27. Langguth B, de Ridder D. Chapter 36 - Tinnitus: therapeutic use of superficial brain stimulation. In: Lozano AM, Hallett M, editors. Handbook of Clinical Neurology: Brain Stimulation. Elsevier (2013). p. 441-67. Available from http://www.sciencedirect.com/science/article/pii/B978044453497200036X

28. Langguth B, Schecklmann M, Lehner A, Landgrebe M, Poeppl TB, Kreuzer PM, et al. Neuroimaging and neuromodulation: complementary approaches for identifying the neuronal correlates of tinnitus. Front Syst Neurosci (2012) 6:15. doi:10.3389/fnsys.2012.00015

Conflict of Interest Statement: The authors declare that the research was conducted in the absence of any commercial or financial relationships that could be construed as a potential conflict of interest.

Copyright $\odot 2017$ Engel, Markewitz, Langguth and Schecklmann. This is an openaccess article distributed under the terms of the Creative Commons Attribution License (CC BY). The use, distribution or reproduction in other forums is permitted, provided the original author(s) or licensor are credited and that the original publication in this journal is cited, in accordance with accepted academic practice. No use, distribution or reproduction is permitted which does not comply with these terms. 